\title{
Subcores in cosmic-ray air showers and high transverse momentum
}

\author{
W E Hazen $\dagger$, A Z Hendel†, A G Ash $\$, J$ M Foster $\ddagger$, B R Green \\ A L Hodson $\ddagger$, M R Porter $\$$ and R M Bull§ \\ $\dagger$ University of Michigan, Ann Arbor, MI 48109, USA \\ ¥University of Leeds, Leeds, UK \\ $\S$ University of Nottingham, Nottingham, UK
}

Received 20 February 1981

\begin{abstract}
The rate of subcores in cosmic-ray air showers has been measured near sea level with a close-packed $35 \mathrm{~m}^{2}$ array of spark chambers at Leeds. A large transition effect was observed from wood beams in the original roof. After the first year, the spark chambers were affixed directly to a new styrofoam sandwich roof of thickness $20 \mathrm{~cm}\left(2.2 \mathrm{~g} \mathrm{~cm}^{-2}\right)$ in order to minimise transition effects. The measured rate is $0.038 \pm 0.01$ per shower of size $N \geqslant 10^{5}$ particles for subcores of size $n_{25} \geqslant 50$, where $n_{25}$ is the net number of subcore particles within a circle of radius $25 \mathrm{~cm}$. The dependence on subcore size goes approximately as $n_{25}^{-3,4}$. The higher rates of subcores reported in other experiments are probably due to transition effects. If $p_{\mathrm{t}}$ is calculated by assuming subcore production by $\pi^{0}$ 's, we find no cases of $p_{\mathrm{t}}>5 \mathrm{GeV} / c$. The problem of abstracting realistic information from the subcore data in order to test theories of high-energy interactions at $10^{15} \mathrm{eV}$ is treated. Simulation results that predict significant improvement in data acquisition at higher altitudes are presented.
\end{abstract}

\section{Introduction}

It will be at least three years before an accelerator (FNAL colliding beam) will displace cosmic rays for the study of nuclear interactions at $10^{15} \mathrm{eV}$ (Jones 1979). Meanwhile, there is a possibility of making significant tests of theories for deep inelastic interactions up to $10^{15} \mathrm{eV}$ from detailed observation of cosmic-ray air showers (Gaisser 1976).

The known products of the deep inelastic interactions are jets of hadrons, which are detected with calorimeters in current accelerator experiments (Bromberg et al 1980 and references therein). In the case of cosmic-ray air showers the air acts as the 'calorimeter'; that is, a jet will produce a sub-shower in the air. The 'calorimeter' is sampled at one depth only, with a spark chamber array, for example. The sub shower may appear as a 'subcore' in the lateral distribution of particles in air, superposed on the main shower distribution.

The belief has been that the frequency of high- $p_{\mathrm{T}}$ events is just great enough to make experiments possible at the low beam intensity of cosmic rays (Gaisser 1976). In fact, the first tentative evidence for an unexpectedly large cross section for high- $p_{\mathrm{T}}$ secondaries came, not from accelerators, but from the pioneering work of the Tokyo cosmic-ray group (Matano et al 1968, 1975), who observed at least 19 subcores of significant size with a $20 \mathrm{~m}^{2}$ spark chamber array in 10000 hours (rate $=10^{-4} \mathrm{~m}^{-2} \mathrm{hr}^{-1}$ ). A Kiel group (Boehm et al 1968) used a hodoscope array of Conversi bulbs and observed 9 subcores in $32 \mathrm{~m}^{2}$ in $5000 \mathrm{hr}\left(0.6 \times 10^{-4} \mathrm{~m}^{-2} \mathrm{hr}^{-1}\right)$, roughly the same rate as Tokyo. Other subcore 
experiments that report $p_{\mathrm{t}}>5 \mathrm{GeV}$ at sea level and at mountain altitude have been performed by methods that make interpretation of the data so uncertain that we have not considered the results further.

We started observations at the beginning of 1977 with the expectation of making a convincing measurement due to the good statistics expected from a $35 \mathrm{~m}^{2}$ spark chamber array with a long running time (Hazen et al 1979). Since our subcore rates are much lower than those of previous work, we believe it is important to report them at this time, before completion of the simulation programs needed for adequate testing of interaction models.

\section{Method}

Limited-current spark chambers $(1 \mathrm{~m} \times 1 \mathrm{~m} \times 2 \mathrm{~cm})$ with glass between the gas and the metal screen electrodes are used in order to give high multiparticle efficiency even for densities greater than 1000 per $\mathrm{m}^{2}$. Two independent arguments and an auxiliary experiment indicate that the spark to particle ratio (efficiency for charged particles) is close to one in this type of chamber. The chambers are in a $5 \mathrm{~m} \times 7 \mathrm{~m}$ close-packed array with an additional eight chambers a few metres from the four edges. All chambers are nearly in contact with the roof and are photographed from below. The roof was originally a composite of wood gutters alternating with fibreglass skylights (run I). This roof was replaced at the end of 1977 by a uniform styrofoam sandwich slab of thickness $20 \mathrm{~cm}$ and mass $2.2 \mathrm{~g} \mathrm{~cm}^{-2}$. The new roof is designed to minimise both the number of subcores produced in the roof and also the enhancement of incident subcores.

The trigger is intended to be at a low enough level so that the effective data cuts can be made during analysis. It is based on a minimum pulse height $\left(5-10\right.$ particles $\left./ \mathrm{m}^{2}\right)$ in a central scintillator in coincidence with one or more particles in nearby scintillators.

The spark chamber photographs were projected to about one-tenth real-space size for scanning and spark image counting. Run I photos were double scanned to permit an estimate of scanning efficiency for subcores, which was found to be approximately $70 \%$ for a single scan.

\section{Data abstraction}

The photographs were scanned for evidence that $(a)$ the centre of a main shower had hit the $35 \mathrm{~m}^{2}$ array, and/or $(b)$ a subcore appeared in the array. The evidence for $(a)$ is a radially symmetric peaking in the particle density (that can be subtle for flat showers). The evidence for $(b)$ is a local peak in density that fades into the background of the main shower at about a metre or less in subcores thus far observed.

Photographs selected in the scanning were then subjected to spark counts, the method depending on the type of event. When only a main shower was involved, the counts were usually taken only at several radial distances from an eye-judged centre of symmetry, in order to determine a shower size $N$ and an 'age' $s$.

For a photograph showing a subcore candidate, the main shower was analysed by taking the particle counts in $20 \mathrm{~cm} \times 20 \mathrm{~cm}$ squares to permit computer fits for best centre of symmetry of the main shower, as well as size and age. Counts for the subcore were taken in rings of width $5 \mathrm{~cm}$ that were eye-centred on the subcore candidate. 


\section{Data reduction}

In order to compare our trigger efficiency with previous work we made a detailed study of the main shower rate in a representative 2100 hours of run. First, NKG functions were fitted to the showers (Nishimura 1966) in the traditional manner. As our most distant detector was never more than 10 metres from a shower core, the shower sizes, $N$, and age parameters, $s$, thus determined were subject to two important biases; firstly the tendency of showers to be relatively flatter near the centre led to overestimates of $s$, and hence of $N$; secondly random errors in determination of $N$, together with the steeply falling $N$ spectrum led to a systematic overestimate of the number of large $N$ 's. This procedure thus gives an upper limit for the size spectrum.

The same sample of showers was then analysed using an average lateral distribution $(s=1.26)$ determined for showers of sizes $2 \times 10^{5}<N<2 \times 10^{6}$ at Kiel (Bagge et al 1979) with a detector which should give a similar response to ours. In this case the fitted density at $r=2.5 \mathrm{~m}$ was simply multiplied by a constant factor of 1430 . This procedure should give a lower limit for the size spectrum because $s$ should be larger for showers with $N<2 \times 10^{5}$ and also the effect of real fluctuations in $s$, together with the steep spectrum, is neglected. From figure 1 we see that the rates are in reasonable agreement with previous work for $N>10^{5}$.

In order to make our comparison of rates with other work conservative, we will use the lower spectrum for our sample.

The main showers in photos that had subcore candidates were additionally fitted by a computer program which first optimised the centre of symmetry, omitting the region of the subcore candidate from the fits. A fit over an appropriate range of radii was used to determine a 'background' to be subtracted from the subcore counts.

The subcore energy, $E_{0}$, and height of production, $h$, were determined in the traditional manner by computer fit of Nishimura-Kidd core approximation functions. The initial

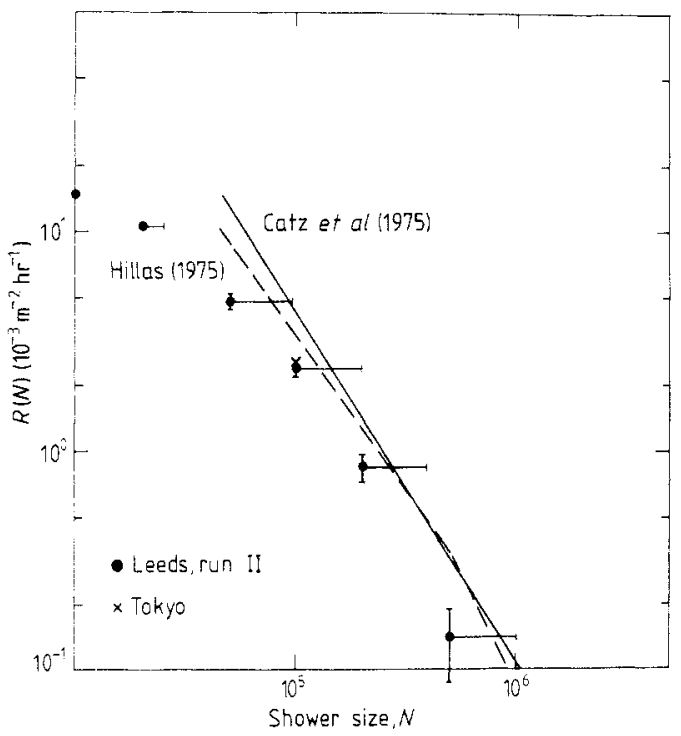

Figure 1. Integral shower size spectrum. The full circles represent the lower estimate of shower size (see text). 
purpose is to have a basis for direct comparison of our observed subcore rate with previous work. The 'size', $n_{25}$, is the net number of particles within a circle of radius $25 \mathrm{~cm}$, obtained from the computer fit. This does not differ appreciably from the result of the direct net count for $n_{25}$.

\section{Observed subcore rate}

The results from run I showed $(a)$ that our roof beams produced a significant transition effect that increases the frequency, confirming the Kiel results, and $(b)$ that we disagreed with Tokyo and Kiel on the frequency of subcores.

From (a) above it is clear that comparisons with other experiments can be difficult if there is significant roof structure above the detectors. The transition effect in roof structure is due firstly to transition of the EM component, which will be particularly important near the axis of subcores, and secondly to interactions by hadrons. In general, we expect both effects to be significant. Because of the complexity of the transition effect, a correction to air is very uncertain for any subcore that appears under a beam. Hence, we utilise only data from subcores that are unlikely to have passed through beams. For our run I, this means restriction to the area under the skylights, which is one-half the total array.

For run II, the full area of $35 \mathrm{~m}^{2}$ was free of beams and the transition effect is believed to be small. (A run with additional wood of $2.2 \mathrm{~g} \mathrm{~cm}^{-2}$, doubling the material, over half the array is being evaluated.)

Our results to date are shown in figure 2 in the form of integral frequency as a function of subcore size, $n_{25}$. No correction has been made for finite detector size or for obscuration near the centre of the main shower. A cut has been made to include only subcores from main shower sizes $N \geqslant 10^{5}$.

For initial comparison, we also show the results of Tokyo and of Kiel. Their subcore sizes were obtained in private communication with Nagano and Samorski respectively. As

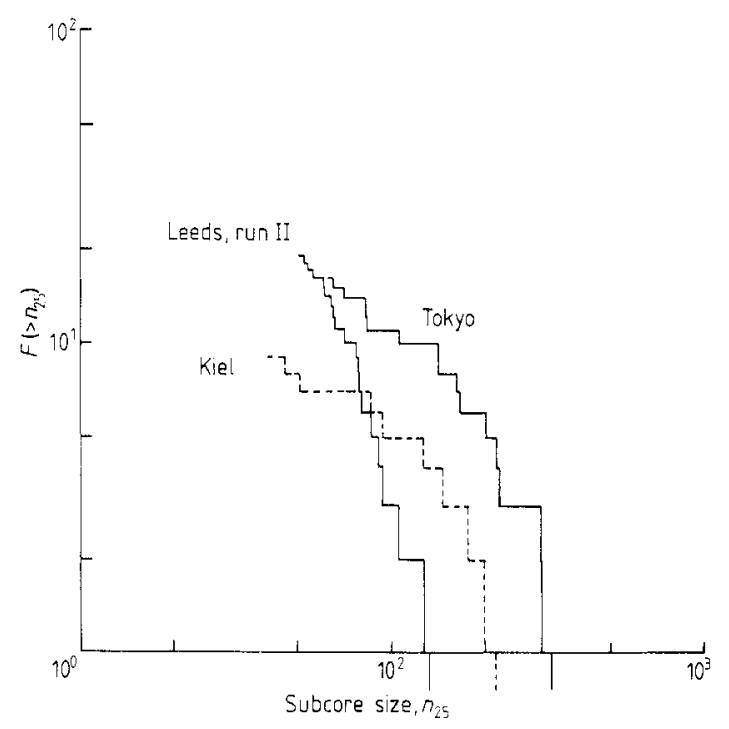

Figure 2. Integral distribution of subcore size $\left(n_{25}\right)$. Area $\times$ sensitive time as follows: Leeds, $5900 \mathrm{hr} \times 35 \mathrm{~m}^{2}$; Kiel, $5000 \mathrm{hr} \times 31 \mathrm{~m}^{2}$; Tokyo, $10000 \mathrm{hr} \times 20 \mathrm{~m}^{2}$. Relaxing the $N>10^{5}$ requirement on the Leeds data adds five subcores with $n \leqslant 60$, and one with $n \approx 100$. 
in our data, there were no corrections for finite detector size or for obscuration. In the case of Tokyo, the cut for main shower size was also $N \geqslant 10^{5}$, but they made additional cuts based on further analysis, namely, height of origin in cascade units, $t \geqslant 6$ and $p_{\mathrm{t}} \geqslant 5 \mathrm{GeV} / c$.

A large proportion (Hazen and Burke estimate 50\%) of the smaller Kiel subcores $\left(n_{25} \leqslant 150\right)$ were believed to originate from local hadron interactions and were cut from the sample. The remaining nine events-presumed 'genuine'-have been reanalysed at Leeds from the photos, in order to make the comparison with our results more direct. Imposition of the cut for $N \geqslant 10^{5}$ has reduced the sample from 13 to 9 subcores.

The gross acceptances (integrated running time $\times$ area) were $2.1 \times 10^{5} \mathrm{hr} \mathrm{m}^{2}$ for Leeds (run II), $2 \times 10^{5}$ for Tokyo, and $1.6 \times 10^{5}$ for Kiel, roughly the same. Another measure of gross acceptance is the number of main shower hits observed within the array. For $N \geqslant 10^{5}$, the number of hits was approximately the same $(\sim 500)$ in the three experiments, indicating similar acceptances. This latter comparison is perhaps better used in another way; it indicates that the three triggers were equally efficient for showers of $N \geqslant 10^{5}$.

Since the acceptances are nearly the same, figure 2 shows that our measured rate of large subcores is much smaller than that of the other experiments. If corrections were made for edge effects, the Tokyo rate would be increased even more relative to Leeds since their array was smaller. There would be a further increase in the disagreement if the $t$ and $p_{\mathrm{t}}$ cuts were not made in the Tokyo subcore data.

\section{Subcores in air}

In the above, observed subcores were discussed. How can we deduce the frequency against size of subcores incident from the air? There are three principal questions to address: trigger efficiency, detector efficiency, and the effect of material above the detectors (transition effect).

The trigger level at Leeds was set low enough so that the efficiency for showers of $N \geqslant 10^{5}$ was high over the entire $35 \mathrm{~m}^{2}$ array. The evidence for this is the uniform distribution of shower axes over the area of the array even though the controlling scintillators were near the array centre. Furthermore, the absolute rate agrees with previous work including that of Tokyo.

The detector efficiency at Leeds (Fukui-type spark chambers, but with tops and bottoms of Georgian-wired glass) is found to be high and independent of particle density up to a limit set by image faintness rather than sparking probability. The same is probably true of the Tokyo spark chambers. Tests for the efficiency of the Conversi bulbs at Kiel showed that it is also high.

Material above the sensitive volume of a detector causes a transition effect due to additional development of the EM component and to hadron production by hadrons. The height of this material above the detector affects what is observed, because of divergence. There has been only sketchy information on the magnitude of the transition effect. It was first observed at Kiel (Boehm et al 1968) as a significantly higher rate of small subcores under about $10 \mathrm{~g} \mathrm{~cm}^{-2}$ of wood than under $2.5 \mathrm{~g} \mathrm{~cm}^{-2}$. It has been noted since then (private communication of Kiel data) that the rate of large subcores at Kiel was also dependent on the material. The observed effect at Leeds (referred to earlier) was of about the same magnitude as at Kiel, namely a factor of at least two increase in rate, for about $10 \mathrm{~g} \mathrm{~cm}^{-2}$ compared with $1 \mathrm{~g} \mathrm{~cm}^{-2}$. Further, the factor appears to increase with subcore size (figure 3). The effect of a uniform layer has just been measured at Leeds (figure 4). It appears that a few $\mathrm{g} \mathrm{cm}^{-2}$ probably give negligible transition effect. 


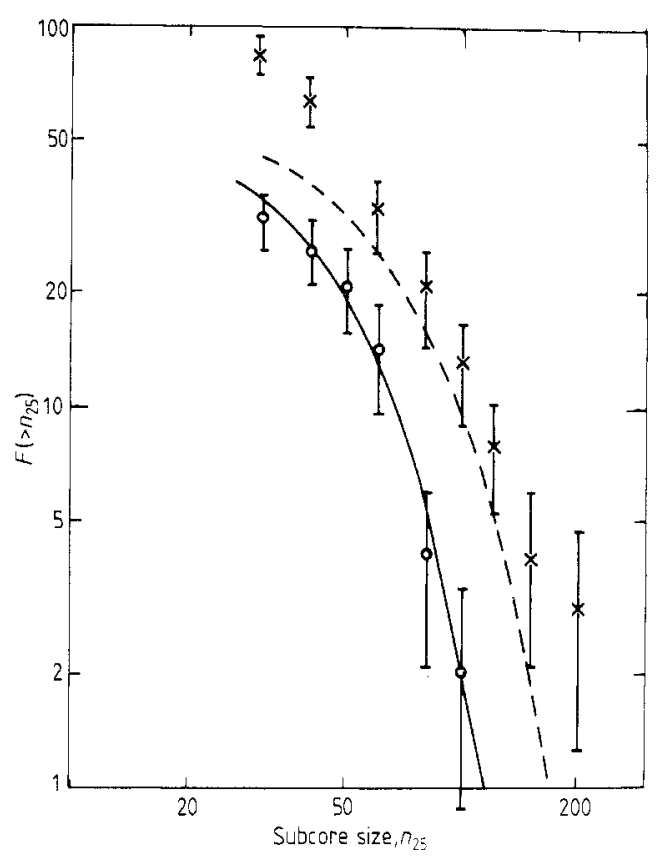

Figure 3. Effect of overlying material on subcore size distributions, from Leeds run I. Beam and skylight areas were equal. The broken curve represents $a(x 1.4)$ transition factor applied to the skylight subcore sizes (Hillas et al 1979). $\times$, broken area $\left(10 \mathrm{~g} \mathrm{~cm}^{-2}\right) ; 0$. skylight $\left(1 \mathrm{~g} \mathrm{~cm}^{-2}\right)$.

In view of the above, we believe that the Leeds data from the area under the skylights in run I and for the entire area in run II give a good approximation to the subcore rate in air. The Kiel data can be similarly treated by using only the area free of the beams, which is about $\frac{3}{4}$ the total area. Only one subcore remains in the sample after the cuts for beams and shower size! The Tokyo array had about $1.5 \mathrm{~g} \mathrm{~cm}^{-2}$ of glass (mirrors) above about $\frac{2}{3}$ of the area, darkroom roof and laboratory roof over the entire array, and four steel girders plus angle-iron rafters supporting the roof. An auxiliary experiment to test the transition effect of steel girders is underway at Leeds. A preliminary run with steel beams approximately 2 feet above the chambers gave a very high rate of 'subcores'. At about 4 feet, the rate had fallen off noticeably, though one very large subcore $\left(n_{25} \simeq 280\right)$ was observed during this 470 hour run. A long run with girders at about 9 feet (the approximate height in the Tokyo experiments) is in progress.

Meanwhile, a conservative approach to reduction of the Tokyo data to air can be made $(a)$ by using only cases where the subcore was free of girders and rafters, and $(b)$ by applying an estimated transition factor for the mirrors. The free area fraction is about $\frac{1}{2}$. From beam effect observations with the 'old roof' at Leeds, we estimate an increase by a factor two in $n_{25}$ for the subcores that passed through a mirror. Plots of the projected positions of the subcores at the plane of the steel girders (from data privately communicated) show correlation with the ridge girder but not with the cross girders. The ridge girder has two steel rafters immediately above it, so it has the highest concentration of steel. Out of the 15 subcores that have direction data, only 3 appear to be clear of steel material and 2 of these passed through a mirror.

Results from other subcore experiments should be examined for contamination from 

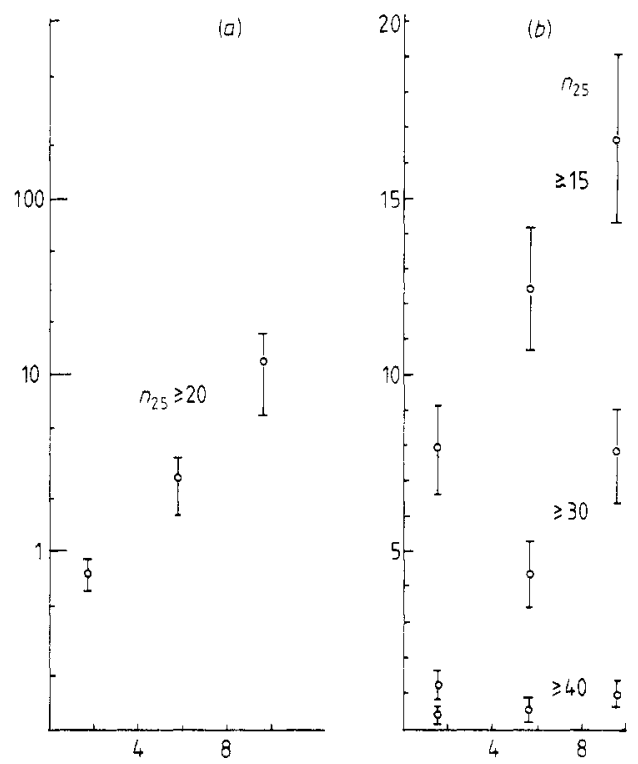

Figure 4. Effect of a uniform layer of wood placed about $30 \mathrm{~cm}$ above the spark chambers. The south half of the array was covered by the 1.75 inch $\left(2.2 \mathrm{~g} \mathrm{~cm}^{-2}\right)$ and 5.75 inch $\left(7.2 \mathrm{~g} \mathrm{~cm}^{-2}\right)$ layers. A central $2 \times 5 \mathrm{~m}^{2}$ strip was covered by the 9.75 inch $\left(12.2 \mathrm{~g} \mathrm{~cm}^{-2}\right)$ layer. Data for larger $n_{25}$ cuts are not shown on the left graph because of frequent zeros for rate under roof alone. Graph $(a)$ shows relative rate (roof with absorber/roof alone) plotted vertically against inches of wood absorber $\left(\simeq \mathrm{g} \mathrm{cm}^{-2}\right)$; graph $(b)$ shows subcore rate under absorber (number per 1000 photographs) plotted vertically against inches of wood absorber $\left(\simeq \mathrm{g} \mathrm{cm}^{-2}\right)$.

transition effect and hadron interaction in overlying material-and, in the case of scintillators, in the detectors themselves. To our knowledge, this has been done only for the results from the Caucasus array (Alexeyev et al 1977) by Olejniczak et al (1979), who concluded that the observed subcores could be entirely attributed to the above effects.

We conclude that, from currently available information, the statistical and transition effect uncertainties for the subcore rate in air at sea level are very large for all previous experiments. Therefore, we believe that our measurement is the first with small enough errors to be truly informative. Our rate for $n_{25} \geqslant 100$ subcores is lower by a factor of two than published results of earlier experiments, and the factor is larger when we attempt to deduce rates in air for the earlier experiments.

\section{Subcore moments}

In addition to subcore size, $n_{25}$, one observes the separation, $R$, of the subcore from the main shower axis of symmetry. The product $n_{25} \times R$ is the best quantity for comparing overall observational results of different experiments because the transverse momentum, $p_{\mathrm{t}}$, derived for a subcore is roughly dependent on the above product. The results for the Leeds data are shown in figure 5 , together with the Kiel and Tokyo results. The measured radial distribution for Leeds $\left(n_{25} \leqslant 50\right)$ subcores is well expressed by an exponential with $R_{0} \simeq 0.5 \mathrm{~m}$. 


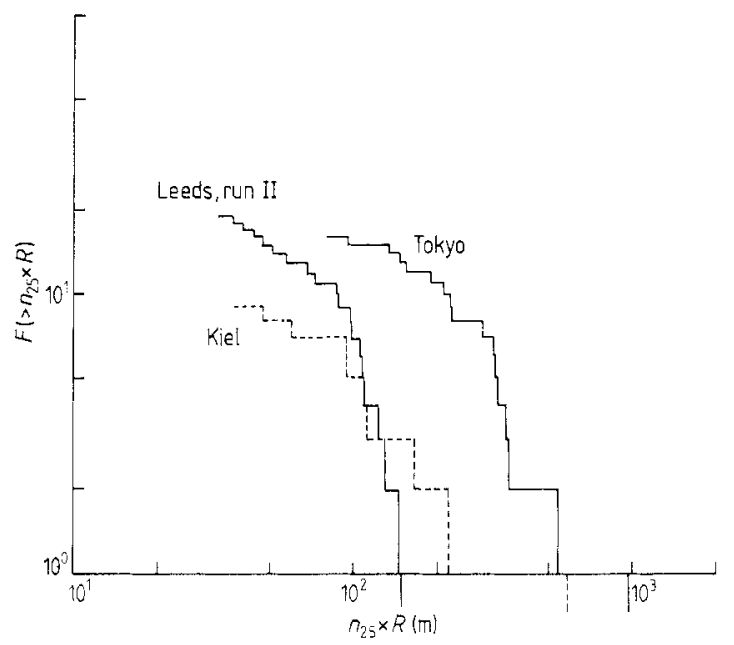

Figure 5. Integral distribution of subcore moments $\left(n_{25} \times R\right)$. The Leeds distribution is somewhat flattened at small $n_{25} \times R$ due to the requirements that $n_{25} \geqslant 50$ and $R \geqslant 0.5 \mathrm{~m}$. The $N>10^{5}$ cut has removed six events all with $n \times R<100$. The other data must be similarly affected.

\section{Subcores per shower}

In order to determine cross sections for interactions that produce subcores, we also need to know the probability per shower. The observed rate must be corrected for finite area of the array and for loss of detectability of subcores against the background of particles of the main shower (obscuration).

The correction for finite area is made by determining the fraction, $\varphi$, of a circle of radius $R$ that falls within the array, where the circle is centred on the main shower and the subcore is on the circle. In run I, sections of the circle that lay under the beam regions were omitted. Then each event was weighted by $1 / \varphi$. The average value of $1 / \varphi$ was about 2 in run $I$ and 1.11 in run $I I$.

The obscuration correction proved to be rather small and therefore the following simplifying assumptions used in the evaluation are satisfactory. The size of background against which a given size of subcore is statistically significant was determined by the Hillas $(1975 a, b)$ method that applies to movable bins. The approximation that the statistics are those of independent particles was found to be valid by a (separate) study of the frequency distribution of particles in azimuthal bins of a given ring in a sample of showers whose axes hit the array but contained no subcores. The radial and size distributions of subcores were approximated by the observed (uncorrected) distributions. Then the loss in observable area near the centres of main showers was calculated. The resulting correction to the probability of a subcore is about $11 \%$ for showers of $N \geqslant 10^{5}$.

After making the above corrections, the calculated probability that a subcore with $n_{25} \geqslant 50$ accompanies a shower of size $N \geqslant 10^{5}$ at sea level is 0.047 . We do not have enough data to justify an attempt to separate out possible dependences on $N, n_{25}$, and $R$.

An early measurement by La Pointe (1962) with an array of 27 fast pulse ion chambers at Michigan gave 9 subcores for 400 showers of $N \geqslant 5 \times 10^{4}$. This rate of 0.02 per shower is consistent with our Leeds rate within the limited accuracy of the old method. The chambers were thin-walled and had no roof over them. 


\section{Uncertainties in the measurements}

In this section we will discuss the uncertainties in the measurements only, and in the next section we will discuss further uncertainties that affect the traditional computation of $p_{t}$. Information on the size of main showers is not needed in the mainstream analysis of the experiment. We evaluate the main shower frequency, spatial distribution of axis positions, etc, principally as a means of determining the useful area of observation and as a means of comparing the size of our observational sample with previous work. However, it is useful to discuss the uncertainties.

The uncertainties in determining the main shower size, $N$, have already been discussed in 'data reduction'. The scanning efficiency for detecting main shower hits on the array has not been tested by double scanning. It is probably high for showers of average steepness and greater, i.e., shower age $s \leqslant 1.3$, but may fall off appreciably for flatter showers. Since the rate of main showers deduced from our data is in substantial agreement with previous results, our scanning efficiency is probably high and/or our method of determining $N$ leads to about the same result as by other methods, in spite of the fact that we do not have auxiliary scintillators outside the array, which would aid in detecting hits by flat showers.

The uncertainty in the main shower to subcore separation, $R$, depends on the uncertainty in position of the subcore axis, which is small, and that of the main shower axis. There are two sources of uncertainty in the true position of the main shower axis: (a) location of the optimum centre of symmetry of the particle distribution, and $(b)$ the deviation of this centre of symmetry from the true axis position, due to the predominance of a few local cascades at observation level. In our experiment $(a)$ is rather small. Distributions of $\chi^{2}$ for shower fits at various trial positions indicate that it is typically $\sim 0.3 \mathrm{~m}$, with a few values up to 1 metre. Shower simulations indicate that errors $(a)$ and (b) together may be of the order of $0.5 \mathrm{~m}$, on average.

\section{Evidence for high $p_{\mathrm{t}}$}

From our spark chamber pictures, we can find the size, steepness, and axis to core separation of each subshower. From these data, $p_{\mathrm{t}}$ is traditionally found in the following way. It is assumed that the subcore is produced by a single (leading) $\pi^{0}$ or gamma ray. The size and steepness of a subshower determine both its energy, $E_{0}$, and its production height ( $t \mathrm{cu}$ or $h$ metres), assuming a purely electromagnetic shower, whose average lateral distribution of particles is given by the Nishimura-Kidd ${ }^{12}$ approximation. Then the transverse momentum, $p_{\mathrm{t}}=E_{0} R / h$ is calculated. We will show later that there are serious difficulties in this analysis but we will first use this method in order to compare our results with those of others.

Our resulting frequency distribution for $p_{\mathrm{t}}$ is shown in figure 6. Shown for comparison are the Tokyo and Kiel distributions. All sets of data are for subcores that occurred in showers of $N \geqslant 10^{5}$. There are no corrections for finite array size or obscuration near the centre of the main shower. The Leeds results are thin roof (run II) only; all Leeds and Kiel events with $t \geqslant 6 \mathrm{cu}$ are included but Tokyo events had an additional cut for $p_{\mathrm{t}} \geqslant 5 \mathrm{GeV} / \mathrm{c}$. The disagreement in $p_{\mathrm{t}}$ frequency is not unexpected in view of the disagreement in observed subcores themselves.

We now turn to the question of uncertainties in the above method for finding $p_{\mathrm{t}}$ in which it is assumed that subcores are due primarily to single $\gamma$ rays. (The results are changed very little if $\pi^{0}$ 's are assumed.) But simulated jets from current QCD (data provided 


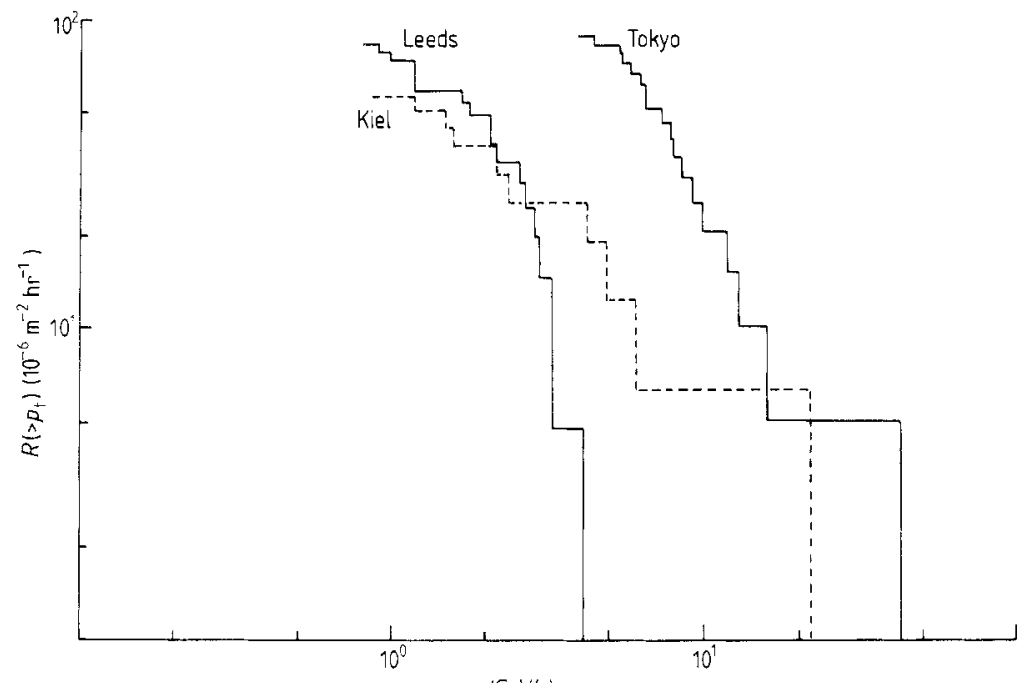

$p+(G e V / c)$

Figure 6. Subcore $p_{\mathrm{t}}$ rates, not corrected for transition effects. The $N>10^{5}$ cut has removed 13 subcores from the Leeds sample all with $p_{-}<2.5 \mathrm{GeV} / c$. An additional cut has been made to include only events with $t \geqslant 6$ cascade units, as with the Tokyo data.

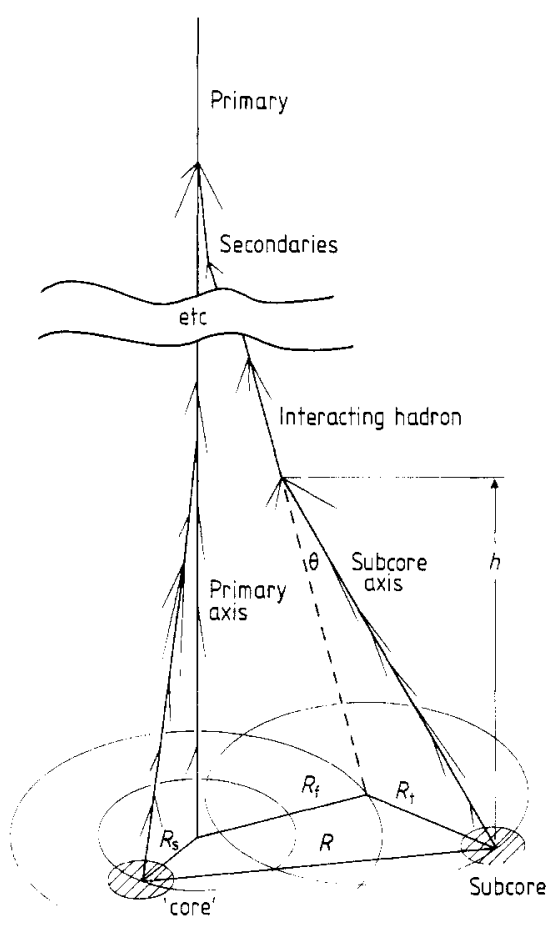

Figure 7. Origin of a subcore in an air shower. See text for an explanation of the symbols. The height of subcore origin $(h)$ is typically $2-5 \mathrm{~km}$, while the primary interaction height is of the order of $15-20 \mathrm{~km}$. 
by I Gaines 1980 , private communication) at $100 \mathrm{GeV}$ CM give low probability for strongly leading $\gamma$ rays or $\pi^{0}$ s. Thus a typical jet would probably give a diffuse subcore that would rarely show up against main shower background. Instead, we would see only the relatively unusual strongly leading $\gamma$ rays or $\pi^{0}$ 's, giving us a bias similar to that arising from single high- $p_{\mathrm{t}}$ particle triggers at accelerators. On the other hand, simulations and experiments on EM cascades (Hotta et al 1979) indicate that fluctuations are large, and that the use of average values lead to large systematic errors in both $E_{0}$ and $h$. The effect of these errors would be to give values of $p_{\mathrm{t}}$ which were very uncertain, and probably too large.

Errors due to the assumption that the angle of emission relative to the direction of the hadron that produced the interaction, $\theta_{\mathrm{t}}$, is well represented by $R / h$ are difficult to assess. The quantity $R$ should be the true separation, $R_{t}$, between the projected 'impact' point of the interacting hadron and the subcore axis, figure 7 .

For the sake of comparison with previous results, we have made the usual approximation that $R_{\mathrm{t}} \simeq R$. This approximation assumes that both $R_{\mathrm{f}}$ and $R_{\mathrm{s}}$ are small compared with $R$, where $R_{\mathrm{f}}$ is the distance of the projection of the interacting hadron from the shower axis and $R_{\mathrm{s}}$ is the departure of the measured symmetry axis of the lateral distribution of particles from the shower axis (figure 7). These questions can only be addressed with simulations. Preliminary results from Leeds simulations indicate that $R_{\mathrm{t}} \simeq R \pm 0.5 \mathrm{~m}$.

Our opinion at this time is that the general method of the above traditional analysis, i.e. starting with observed results and working back to find an average parent interaction, can give only very rough approximations. Instead, we plan to carry simulations through from the primary to predicted subcore distributions. The models will be tested by comparison of simulation results with the observations.

\section{Predictions for high-altitude observations}

The frequency of subcores under thin roofs at sea level has proved to be disappointingly low. Since the intensity of the hadron 'beam' that interacts to produce the generators of the subcores increases rapidly with altitude, the rate of observed subcores should increase correspondingly. We have an estimate of the expected magnitude of the increase from Leeds simulations for primaries of $10^{15} \mathrm{eV}$. A good measure of likely increase in subcore rate is the increase in interactions by hadrons of $E \geqslant 10^{14} \mathrm{eV}$ in the atmospheric layer from 300 to $500 \mathrm{~g} \mathrm{~cm}^{-2}$ above the observation level. (This is roughly the principal source layer for subcore parents, judging from traditional analysis for subcore origin.) The results of the simulations are an increase from sea level $(a)$ by a factor 10 , in going to $3000 \mathrm{~m}$, and $(b)$ by a factor $70 \mathrm{in}$ going to $5500 \mathrm{~m}$. The rate of observed subcores at Mount Norikura $(2800 \mathrm{~m})$ (Sasaki et al 1979) is indeed higher than at sea level by a factor even larger than 17, but it is very difficult to assess the magnitude of the correction to open air for the Norikura data due to the roof effects. An early Michigan experiment (Davis 1954) at approximately $3000 \mathrm{~m}$ gave 6 subcores with $R \geqslant 50 \mathrm{~cm}$ in 48 showers under a thin roof. This gives a factor 4 compared with sea level, but the statistics are poor and the spatial resolution was low. Data from Chacaltaya $(5200 \mathrm{~m}$ ) (Shibata et al 1968) are sparse but suggestive. However, correction to open air is again difficult, because of copper shielding, wood framing, and a steel tower above the spark chambers.

Since the distance between the primary cosmic-ray interaction and the subcore source layer is less at mountain altitude (about $300 \mathrm{~g} \mathrm{~cm}^{-2}$ at $3000 \mathrm{~m}$ in comparison with about 
$600 \mathrm{~g} \mathrm{~cm}^{-2}$ at sea level) both $R_{\mathrm{s}}$ and $R_{\mathrm{t}}$ should be smaller (figure 7) and the determination of $p_{\mathrm{T}}$ more precise.

We conclude that it would be very useful to make subcore observations with a spark chamber array under a thin roof at several mountain altitudes in order to test models for high $p_{\mathrm{t}}$ production at laboratory energies of about $10^{14} \mathrm{eV}$.

\section{Acknowledgments}

This work was supported by the British SRC, the US DOE, the Jersey Education Department and the respective physics departments.

\section{References}

Alexeyev E N et al 1977 Proc. 15th Int. Conf. on Cosmic Rays, Plovdiv 856

Bagge E R, Samorski M and Stamm W 1979 Proc. 16th Int. Conf. on Cosmic Rays, Kyoto 13260

Boehm E, Buscher R, Fritze R, Roose U J, Samorski M, Staubert R and Trumper J 1968 Can. J. Phys. 46 S4 1

Bromberg C et al 1980 Nucl. Phys. B 1711

Catz P, Gawin J, Grochalska B, Hibner J, Hochart J P, Milleret G, Stanczyk J and Wdowezk J 1975 Proc. 14th Int. Conf. on Cosmic Rays, Munich 124329

Davis W P, Hazen W E and Heinemann R 1954 Nuovo Cimento 12233

Gaisser T K 1976 th Int. Coll. on Multiparticle Reactions, Tutzing

Hazen W E and Burke D L 1977 J. Phys. G: Nucl. Phys. 3715

Hazen W E, Hendel A Z, Foster J M, Green B R, Hodson A L, Porter M R and Bull R 1979 Proc. 16th Int. Conf. on Cosmic Rays, Kyoto 8230

Hillas A M 1975a Proc. 14th Int. Conf. on Cosmic Rays, Munich 93439 1975b Phys. Rep. 2061

Hillas A M, Hazen W E, Hendel A Z, Foster J M, Green B R and Hodson A L 1979 Proc. I6th Int. Conf. on Cosmic Rays, Kyoto 8236

Hotta N et al 1979 Proc. I6th Int. Conf, on Cosmic Rays, Kyoto 737

Jones L W 1979 Proc. Air Shower Workshop, Utah ed T K Gaisser (Utah: Bartol Foundation, University of Delaware)

La Pointe M H 1962 PhD thesis University of Michigan

Matano T, Machida M, Ishizuka T and Ohta K 1975 Proc. 14th Int. Conf. on Cosmic Rays, Munich 82971

Matano T, Nagano M, Shibata S, Suga K and Tanahashi G 1968 Can. J. Phys. 46 S56

Nishimura J 1966 Handb. Phys. 46/II

Sasaki H et al 1979 Proc. 16th Int. Conf. on Cosmic Rays, Kyoto 8190

Shibata S, Saavedra O, Kamata K, La Pointe M, Rennex B, Uchino K, Nagano M and Suga K 1968 Can. J. Phys. 46560

Olejniczak J, Wdowczyk J and Zujewska E 1979 Proc. 16th Int. Conf. on Cosmic Rays, Kyoto 8283 